\title{
The international office worker of the future
}

\author{
P. Yoong, Victoria University of Wellington, New Zealand, Chair \\ G. de Vreede, Delft University of Technology, The Netherlands \\ J. Gricar, University of Maribor, Slovenia \\ L. Jessup, Indiana University, US A
}

\section{INTRODUCTION}

In the International Office of the Future (IOF), individuals will use a variety of technologies to assist them in communicating, in collaborating, and coordinating their activities across distance and time. These technologies are designed to enhance workflow automation, office conferencing and communications, information filtering, shared calendaring, electronic meeting support, data interchange, videoconferencing, etc.

Within the networked organization, dual structures will be put in place to manage structured and unstructured work. Business processes would thus need to be easily understood, analysed, diagnosed, and adapted. Problem solving and decision making will be done by ad hoc project teams made up of individuals from several cultural and national backgrounds, with each team member choosing their preferred location and time of work.

As there is great social and cultural diversity across the globe, the IOF also presents many potentially interesting opportunities for discovering and managing our differences and, subsequently, for enhancing global communications, collaboration, and coordination. For example, an individual from one country is likely to encounter difficulties in collaborating with someone from another culture. How things get done in the project teams is governed not only by the project requirements but by differences in languages, cultural norms, values, and expectations. If this crosscultural collaboration is mediated by computer and communications technology, the difficulties become more complex.

As the IOF is expected to be a complex environment, characterised by turbulence and rapid change, in which individuals are expected to adopt a flexible, collaborative, and open-minded approach to their global work, we must start to find what 'works' and what doesn't as we proceed to create an IOF environment. 


\section{PURPOSE /AIM}

The aim of this panel discussion is to explore the opportunities, challenges and issues of the IOF and how these would impact on the role of the International Office Worker of the Future.

\section{FORMAT AND QUESTIONS}

The final IOF multi-site conference in September 1997 has chosen the following three themes as the focal points for discussions: (a) technology, (b) business processes and (c) organizational, cultural and social aspects.

Using each of the three themes in turn, the panel will examine, among other things, the following questions:

1) What opportunities, challenges and issues of the IOF would impact on the role of the International Office Worker of the Future (IWOF)?

2) What is the likely impact?

3) What skills and knowledge do IWOF need in order to manage these impacts, and how can these skills and knowledge be acquired? 